\title{
Clinical Significance of Pulsatile Index and Diastolic Blood Flow for Popliteal and Tibial Bypass Patency
}

\author{
Mihail Cheshmedzhiev ${ }^{1 *}$, Krasimir Ivanov², Iskra Mircheva ${ }^{3}$ and Emil Jordanov ${ }^{1}$
}

${ }^{1}$ Department of Vascular Surgery, St. Marina University Hospital, Varna, Bulgaria

${ }^{2}$ Department of General and Operative Surgery St. Marina University Hospital, Varna, Bulgaria

${ }^{3}$ Department of Social Medicine and Healthcare Organization, Medical University, Varna, Bulgaria

\begin{abstract}
Aim: To compare data from pulsatile index [PI) and diastolic blood flow [DF\%) after local administration of prostaglandines in failed and patent infrainguinal autovenous bypasses for at least one year, as well as to compare the data obtained from the tibial and the popliteal [above and below knee) bypasses and to estimate its clinical significance for their patency.

Patients and Methods: Prospectively for two-year period 107 patients with peripheral arterial occlusive disease, who were subjected to autovenous bypass below the inguinal ligament were included. Intraoperative flowmetry was performed with a VeriQ flowmeter and perivascular probes 'Medi-Stim' at the target artery underneath the distal anastomosis. The measurements were performed after bypass declamping and after intragraft prostaglandin infusion.

Results: Intraoperative measurement of blood flow by means of transit time and prostaglandin application was performed in all 107 patients. There were no significant differences between tibial and popliteal bypasses after prostaglandin administration, which to determine their different patency. Patients with early bypass failure had no significant alteration in the estimated values after prostaglandin infusion. Pulsatile index over 2 by specificity $84 \%$ and DF\% under $51 \%$ by specificity $73 \%$ after prostaglandins are considered unsatisfying.

Conclusion: Prostaglandin application underneath the distal anastomosis results in arterial vasodilatation and is an indicator of the quality of the byass, the anastomoses and run-off arterial capacity. Being dependent on the resistance of the blood outflow, the PI and DF\% are important factors for the bypass prognosis but it cannot be calculated as a certain prognostic factor.
\end{abstract}

Keywords: TTFM; Intraoperative flowmetry; Pulsatile index; Diastolic blood flow; Tibial and popliteal bypasses; Run-off; Prostaglandins

\section{Introduction}

According to the statistics $20-40 \%$ of the bypasses are occluded within 5 years after the operation. Early occlusions are registered in $15 \%$ of the patients [1-3]. The most commonly encountered causes for early bypass failure could be technical (e.g. improperly destroyed venous valves, graft stenoses, anastomotic stenoses) and functional in terms of blood outflow [4]. Its measurement by the means of transit time offers a possibility for a real time intraoperative assessment resulting in bypass correction when necessary.

The hemodynamic success concerns the entire limb for which reason distal sites are used for monitoring. The run-off status after surgical and endovascular revascularization is of major importance for the primary and secondary patency and limb salvage $[5,6]$. There is a wide consensus about the role of the vascular status distally to reconstruction area for the proper graft function [7,8]. Every bypass has a critical run-off. A flow smaller than the critical flow leads to thrombosis [9]. Adequate distal outflow is tantamount to the functional success of vascular surgery and to a patient's immediate recovery. This surgical success is facilitated when quantitative hemodynamic effects can be considered during vascular surgical management.

The transit time flow measurement [TTFM] is a simple and costeffective technology that helps reduce postoperative ischemia and hence, morbidity and mortality. The transit time technique offers many advantages. Measurements are theoretically independent of internal or external vessel diameter, vessel shape, and Doppler angle
[10]. TTFM is also insensitive to the alignment between the probe and the vessel. The probe does not have to be in direct contact with the vessel, and calibration is not necessary. The recordings are stable and data storage and analysis are routinely performed. Many of these features are not offered by the Doppler technology. For this reason, TTFM has become the most widely used device for accurate intraoperative interpretation of graft patency [11]. Clinical experience has shown that absolute flow values per se are not a good indicator of the quality of the anastomoses and cannot justify graft revision by themselves. There are too many variables influencing absolute flow, including size of the graft and quality of the revascularized artery. Moreover, artery flow reserve can better facilitate correct diagnoses of anastomotic imperfections than can absolute flow. Ascer et al. $[12,13]$ have documented that the quality of the anastomosis and run-off artery can be better defined by testing its dynamic ability to increase graft flow using papaverine or prostaglandins. Prostaglandins are usually used to improve the blood flow in patients with end stage peripheral arterial disease $(\mathrm{PAD})$ in the form of i.v. infusion but can also be applied

${ }^{*}$ Corresponding author: Mihail Cheshmedzhiev, Department of Vascular Surgery, St. Marina University Hospital, Varna, Bulgaria, Tel: 0359888982431; E-mail: mi6046@gmail.com

Received March 21, 2015; Accepted April 06, 2015; Published April 08, 2015

Citation: Cheshmedzhiev M, Ivanov K, Mircheva I, Jordanov E (2015) Clinical Significance of Pulsatile Index and Diastolic Blood Flow for Popliteal and Tibial Bypass Patency. J Vasc Med Surg 3: 199. doi:10.4172/2329-6925.1000199

Copyright: () 2015 Cheshmedzhiev M, et al. This is an open-access article distributed under the terms of the Creative Commons Attribution License, which permits unrestricted use, distribution, and reproduction in any medium, provided the original author and source are credited. 
intraoperatively as intraarterial infusion. For a given period of time that results in a better blood flow, distal arterial vasodilatation and the vasospasm is successfully overcome. Prostaglandins are known as tissue hormones which are synthesized and released as a result of irritation and/or damage of the cell membrane and act as vasodilatators due to precapillary sphincter relaxation. They also antagonize the vasoconstrictive action of Thromboxane and Leucotriens.

During clinical experience, D’Ancona G et al. [14] developed progressive expertise in TTFM findings interpretation. Specific rules were developed to correctly interpret the different TTFM findings including diastolic blood flow, pulsatility index (PI), and mean flow values and can be related to one another by the graft patency triangle. Endothelial lesions during vascular surgery, the anastmoses and the gradient of blood flow tangential pressure (shear stress) are the major reasons for the development of intimal hyperplasia [8], leading to stenosis around the anastomoses. Wall shear stress [5] is related to flow and inversely related to diameter. Further progression of the disease, changes in the size of the flow and intimal hyperplasia are the major causative factors for development of thrombosis in the bypass during late postoperative period. This study aims to assess the clinical significance of the pulsatile index and diastolic blood flow for peripheral bypass patency by testing dynamic ability to increase graft flow using prostaglandins, as well as to compare the data obtained from tibial and popliteal (above and below knee) bypasses and to estimate its clinical significance for their patency.

\section{Patients and Methods}

Prospectively for two-year period 107 (99 men and 8 women, mean age 66 years, range $45-83$ years) patients with peripheral arterial occlusive disease, who were subjected to autovenous bypass below the inguinal ligament and intraoperative flowmetry below the distal anastomosis before and after continuous (at least $5 \mathrm{~min}$.) infusion of Prostavasin or Ilomedin were included. All bypasses are made with translocated nonreversed vein.

Risk factors include smoking (39\% of the patients), diabetes mellitus (41\%), hypertension (38\%), ischaemic heart disease $(31 \%)$, cerebro vascular disease (18\%), chronic obstruction pulmonary disease (3\%) and preliminary vascular reconstructions and amputations (46\%). Eleven patients were operated after coronary revascularization and hemodynamics remained stable after local infusion of prostaglandins. We compared the intraoperative PI and DF\% data for all failed autovenous bypass grafts in the order of the time of their diagnostic (32 with thromboses of the graft and 21 with stenoses of the distal anastomosis) with the flowmetry results for 54 patients with patent autovenous bypass grafts in the order the latter were made and that had been patent for at least 12 months (Figure 1). Thirty-nine patients were in IV stage and forty-seven patients had rest pain, while twentyone patients were operated in II b stage. Control examinations were performed regularly on monthly basis and included duplex ultrasonography and measurement of the ankle-brachial index.

Patients with unstable haemodynamics, heart failure, arrhythmia, and impaired diuresis were excluded from the study, due to contraindication for prostaglandin (PG) administration, as well as the patients who attended irregularly the control examinations for objective and subjective reasons and the patients who passed out. Patients with stenoses, who refused corrective procedures but later were found to have thrombosis were considered as patients with thrombosed bypass grafts
Distal anastomoses were made on the popliteal artery above the knee (P1) in 28 reconstructions, below the knee in P3 segment in 39 reconstructions and in the tibial arteries - in 40 bypass graftings. To make a distal anastomosis, the posterior tibial artery was used in 21 reconstructions, the anterior tibial artery - in 14 reconstructions, and the fibular artery - in 5 reconstructions (Figure 2). The intraoperative measurement of blood flow was performed with a VeriQ flowmeter and perivascular probes "Medi-Stim". The first measurement was performed after bypass declamping. The flow probe was localized immediately under the distal anastomosis. The flow volume was registered and then PG were administered as a continuous infusion simultaneously with measuring the flow. Pulsatile index qualifies the pulsativity of the wave form [15] and is used as an indicator of resistance to outflow. Initially this index was used by Gosling in 1971 [16]. Because it was extremely complex, today we use a simplified version:

$$
\mathrm{PI}=\frac{Q \max -Q \min }{Q \text { mean }}
$$

where $\mathrm{Q}$ max is the maximal systolic flow, $\mathrm{Q}$ min is the minimal diastolic flow and $\mathrm{Q}$ mean is the mean blood flow volume. The flowmeter calculates it automatically and the result is visualized in the right upper corner of the screen. Some authors believe that DF\% is very important when we try to define the quality of the bypass [17]. Using ECG synchronization the DF\% is defined as ratio between the diastolic volume and the common volume within one heart cycle.

$$
\mathrm{DF}(\%)=\frac{\int \mathrm{DF}}{\int \mathrm{DF}+\int \mathrm{SF}}-\text { blood flow in the run-off artery during }
$$

diastole

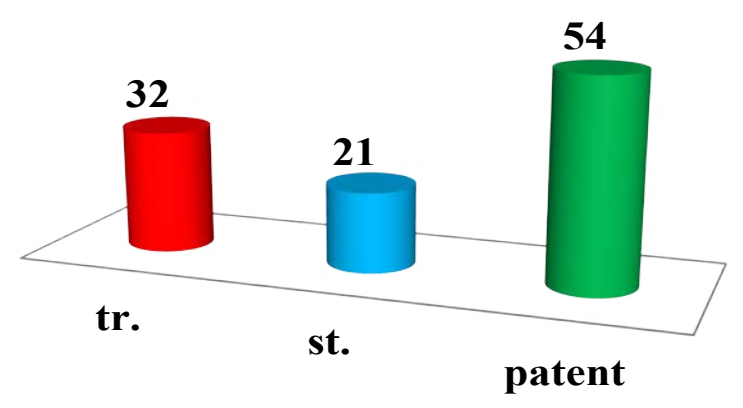

Figure 1: Patients with thromboses of the graft (tr.), with stenoses of the distal anastomosis (st.) - the group of failed bypasses and the group with patent autovenous bypass grafts for at least 12 months.

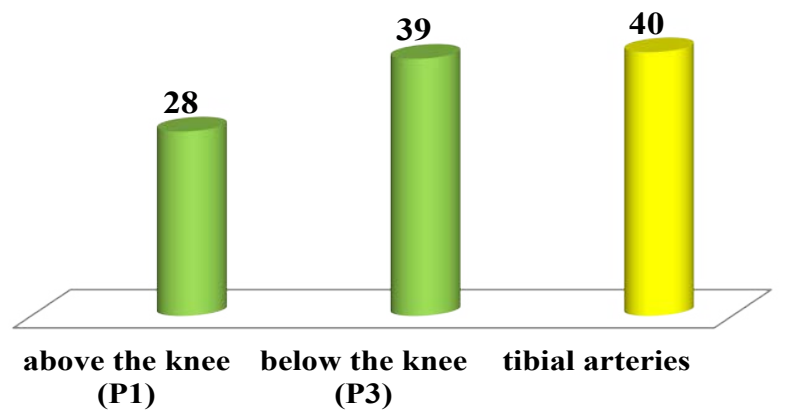

Figure 2: Both groups tibial (yellow) and popliteal (green) bypasses. 
It is displayed under the PI at the screen. Using the transit time principle the blood flow volume is measured directly without additional information about the angle of the ultrasound waves or blood vessel diameter. The principle is based on the fact that longer time is necessary for the ultrasound to penetrate the blood along the blood flow direction compared to the time for penetration in the opposite direction. Both ultrasound crystals are located at the same side of the blood vessel while a reflector stays at the opposite side. The ultrasound is transmitted upward the blood flow from the first crystal and after a period of time t1 it reaches the second crystal. After reversal of crystal function the t2 transit time is measured. The transit time $\mathrm{t}=\mathrm{t} 2-\mathrm{t} 1$ is proportional to the blood flow volume. The results were analysed with SPSS v.12 and STATISTICA. The means and standard deviations were calculated. The Kruskal-Wallis test, ANOVA and the receiver operating characteristics curves (ROC) were used to compare variables before and after druginduced vasodilation in both groups of bypass grafts (patent and failed) The area below the curve was determined by non-parametrical methods; the level of significance was set at $\mathrm{p}=0.0001$.

\section{Results}

We used Kruskal-Wallis test for comparison of 2 parameters and for perspicuity the separate combinations are showed with ANOVA the correlation between PI before and after prostaglandin vasodilatation in tibial and popliteal (above and below the knee). In the group of tibial bypasses (in red) the PI reduction after prostaglandin application is statistically significant $(t=13,730 ; p=0,0005)$. In the group of popliteal bypasses the PI reduction is also statistically significant $(t=9,665$; $\mathrm{p}=0,0001)$. The difference of the PI before vasodilatator application between both groups is statistically insignificant $(t=1,527 ; p=0,13)$ as well as after its application $(\mathrm{t}=1,937 ; \mathrm{p}=0,056)$ (Figure 1$)$.
By means of ROC, we assessed the specificity of the results of the measured PI in failed and patent bypasses after prostaglandin infusion (Figure 2). We received $84 \%$ specificity by optimal PI value -2 as a point, over which after vasodilatation the result would be unsatisfying. The area below the curve was determined by non-parametrical methods. We compared the DF\% data and found a significant increase in tibial and popliteal bypasses after vasodilatator application. In the group of tibial bypasses (in red) - $t=6,93 ; \mathrm{p}=0,0189$ and in the group of popliteal bypasses $-t=14,48 ; p=0,0001$. It is to note that the difference between the basic values in both groups before medication application is significant $(t=2,93 ; p=0,004)$ (Figure 3 ) while after its application there is no significant difference $(t=0,045 ; p=0,964)$. The difference in basic values before vasodilatation is significant $(t=2,93$; $\mathrm{p}=0,004$ ), while no significant difference between values in both groups after PG application was registered $(t=0,045 ; p=0,964)$ - insignificant difference. We used ROC to assess the specificity of the measured DF\% after medication infusion in failed and patent bypasses (Figure 4). We received $73 \%$ specificity by optimal value $-51 \%$ as a point, under which after vasodilatation the result would be unsatisfying. The area below the curve was determined by non-parametrical methods. In the first 30 days within the followed-up patients seven early occlusions were detected. Those patients were observed with minimal decrease of PI (remaining up to 4 or even 5) and minor increase of DF\% (mean 7,5\%) after PG administration compared to the rest (Figures 5 and 6).

\section{Discussion}

The optimal blood flow for each bypass is determined by good inflow, well-constructed conduit and anastomoses and sufficient outflow. Endothelial lesion during vascular surgery and the gradient of blood flow are the major reasons for the development of intimal

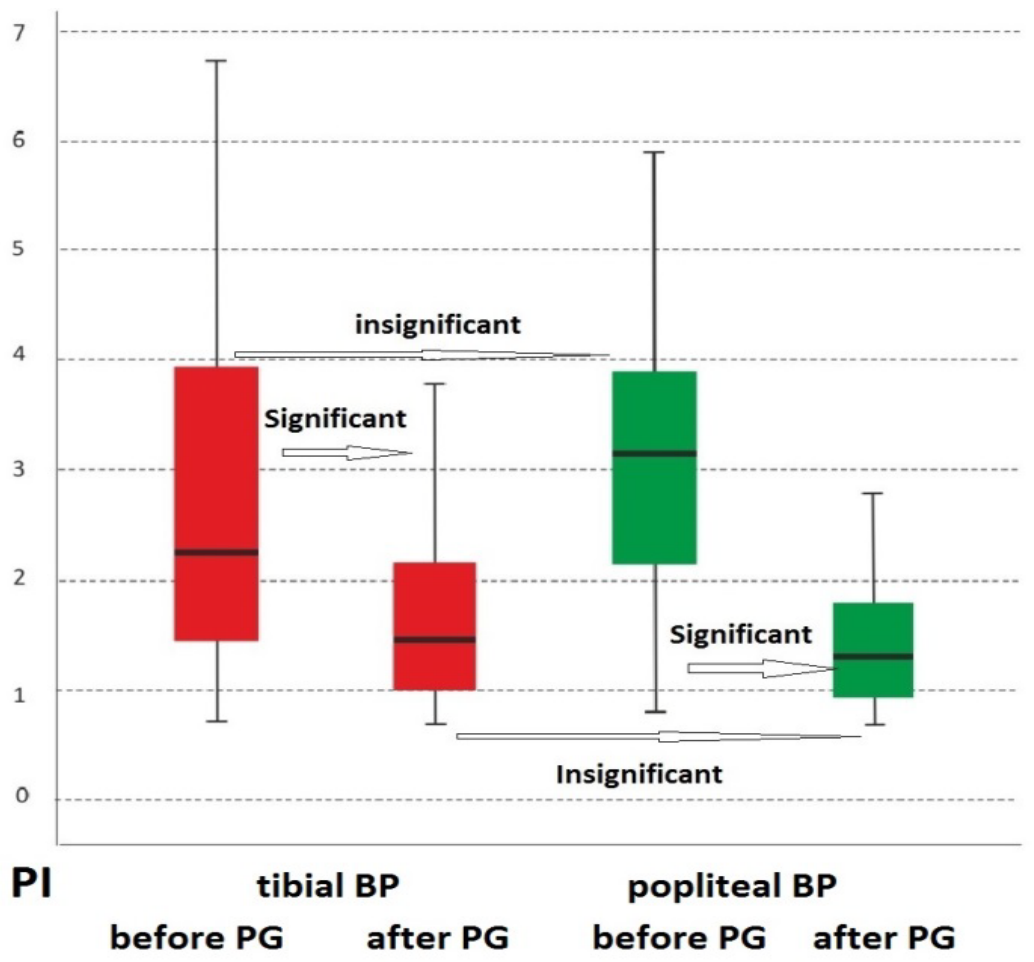

\begin{tabular}{|l} 
마ian \\
$\square$ 25\%-75\% \\
I Non-Outlier Range
\end{tabular}

Figure 3: Correlation of PI before and after medication vasodilatation in tibial and popliteal bypasses (BP). The difference in values before medication application (between the basic values in both groups) is insignificant $-t=1,527 ; p=0,13$, as well as after its application $-t=1,937 ; p=0,056$. 


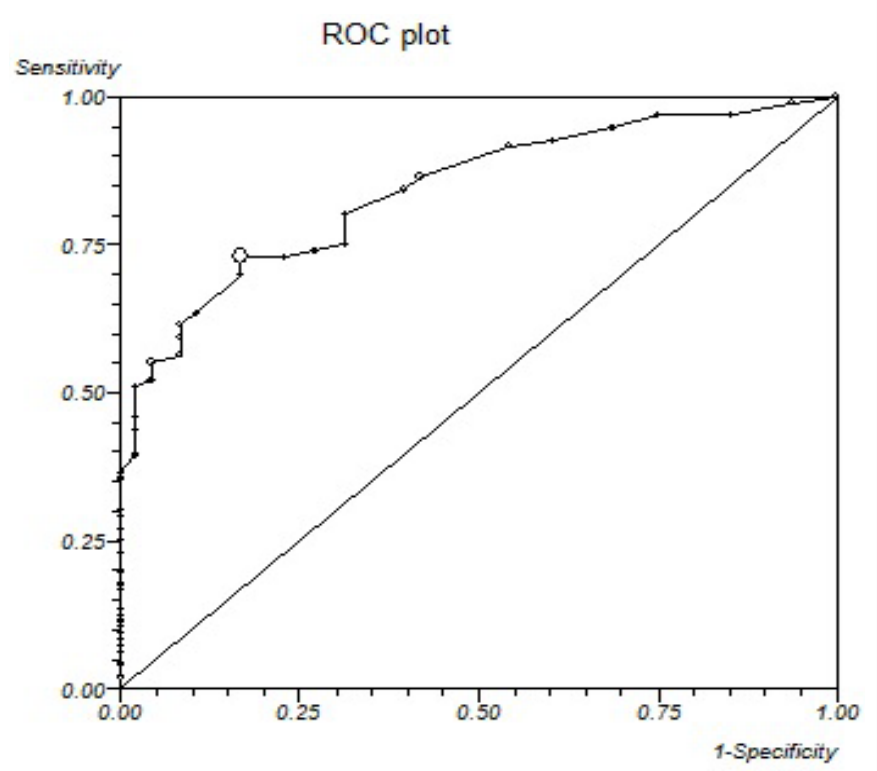

Figure 4: ROC curve analysis $\mathrm{PI}$ before $\mathrm{PF}(+\mathrm{ve}), \mathrm{PI} F$ after (-ve); $\mathrm{AUC}=0$, $8417 ; 95 \% \mathrm{Cl}: 0.7780 .905$; Optimal value (cut-off point) $=2$.

hyperplasia leading to stenosis around the anastomoses and bypass thrombosis [8]. The run-off artery flow measured at the operating table after declamping is normally low than that in a wakeful patient, resulting from the vessel constringent effect of the surgical exposure, the ischemic condition and reperfusion. This constraint is compensated by measuring the flow after stimulation with a vasodilatation agent [18].

Pulsatile index and DF\% are indicators of the quality of the anastomoses and the run-off arterial capacity. It is commonly encountered for the operator to find unsatisfying blood flow immediately after declamping the bypass [19]. After ruling out technical problems (e.g. undestroyed valves in the venous graft, graft stenoses, anastomotic stenosis) a common cause for the reduced blood flow is a vasospasm. Prostaglandin application result in such cases vasodilatation and the operator can measure the artery flow reserve underneath the bypass [18]. In patients with early occlusion minimal decrease of PI (remaining up to 4 or even 5) and minor increase of DF\% [mean 7,5\%] after PG injection in comparison with the rest patients were found, which implies a revision of the newly constructed bypass at the same operative time.

Decrease of PI (Figure 1) and increase of DF\% (Figure 3) after administration of PG in patients with popliteal and tibial reconstructions have no statistically significant difference, which could determine a better outcome for one and worse for the other reconstruction, which shows the importance of the unaffected by the disease distal arterial segment, giving optimal outflow. Significantly lower DF\% before prostaglandine infusion in popliteal bypasses and later insignificant difference with tibial reconstructions after PG administration is connected with spasm followed by vasodilatation of larger cross-section of the arterial segment, which also refers to PI, but there is no such significant difference. Probably DF \% is more sensitive hemodynamic parameter than PI, but we have not seen yet similar suggestion in the literature. Therefore it is necessary to follow more patients to express such a claim. Pulsative index is used mainly in duplex sonography of transplanted organs and for analysis of disease in peripheral vessels. Increase of PI means impairment of the outflow zone i.e. transplanted organ rejection or a stenosis of the arterial flow of the lower limbs. In the literature there are no reports which use intraoperative obtained PI as factor for prediction of patency of femoro-popliteal bypasses.

Using ROC analysis we demonstrated that PI over 2 by specificity

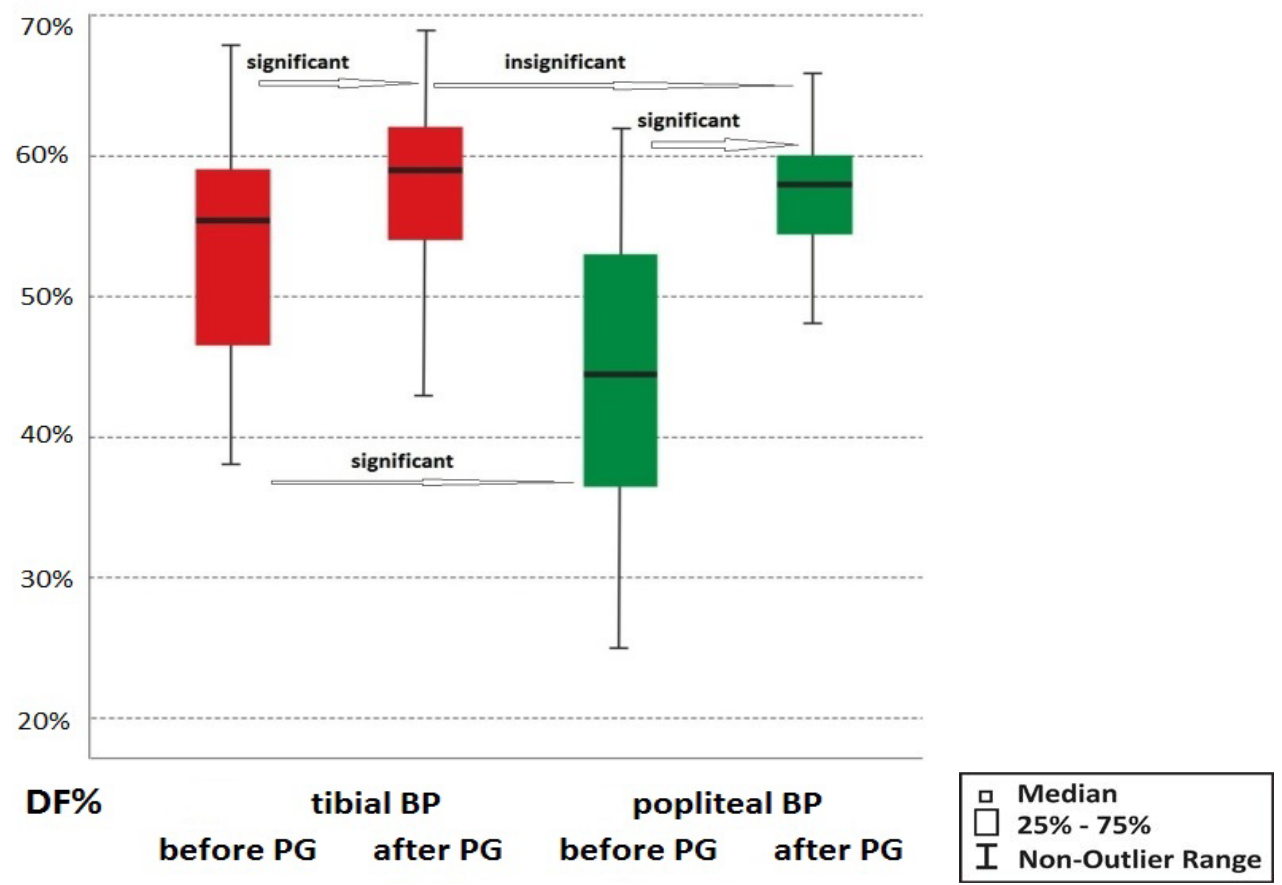

Figure 5: Correlation of DF\% before and after medication dilatation in tibial and popliteal bypasses (BP). 
Citation: Cheshmedzhiev M, Ivanov K, Mircheva I, Jordanov E (2015) Clinical Significance of Pulsatile Index and Diastolic Blood Flow for Popliteal and Tibial Bypass Patency. J Vasc Med Surg 3: 199. doi:10.4172/2329-6925.1000199



Figure 6: ROC curve analysis: DF\% before CF(+ve), DF\%F after (-ve); AUC = 0,73; 95\% Cl: 0.550 0.912; Optimal value (cut-off point) $=51(\mathrm{DF} \%)$.

$84 \%$ and DF\% under $51 \%$ by specificity $73 \%$ are considered unsatisfying. Those reconstructions require intraoperative bypass correction and if the result is still unsatisfying the patients should be closely monitored during the follow up period. The effect of vasodilating substances has no significant prognostic value. The significant PI reduction and DF\% increase have prognostic value to a certain extent but it cannot be calculated as a certain prognostic factor. We cannot deny their role as predictors of the immediate outcome of the operation as we know that extremely high PI and low DF\% are bad prognostic factors [20].

Nevertheless, if high PI (above 2) and low DF\% (under 51\%) is observed after prostaglandin administration, the reason should be found out and corrected to increase DF\% and decrease PI. The unsatisfactory effect after vasodilation medication application must lead the operator to seek technical failure and/or inappropriate level of distal anastomosis. When no possibility for intraoperative correction exists, one must consider a broad postoperative therapy and close follow up of the patients.

\section{Conclusion}

Prostaglandin application underneath the distal anastomosis results in arterial vasodilatation and is an indicator of the quality of the bypass, the anastomosis and run-off arterial capacity. Being dependent on the resistance of the blood outflow, the significant PI reduction and $\mathrm{DF} \%$ increase have prognostic value to a certain extent but it cannot be calculated as a certain prognostic factor. Intraoperative measurement of flow parameters may avoid early bypass occlusion, post-surgical tests, and provide information for further patient treatment during recovery and thereafter.

\section{References}

1. Bergamini TM, George SM Jr, Massey HT, Henke PK, Klamer TW, et al. (1995) Intensive surveillance of femoropopliteal-tibial autogenous vein bypasses improves long-term graft patency and limb salvage. Ann Surg 221: 507-515.
2. Conte MS, Belkin M, Upchurch GR, Mannick JA, Whittemore AD, et al. (2001) Impact of increasing comorbidity on infrainguinal reconstruction: a 20 -year perspective. Ann Surg 233: 445-452.

3. Shah DM, Darling RC 3rd, Chang BB, Fitzgerald KM, Paty PS, et al. (1995) Long-term results of in situ saphenous vein bypass. Analysis of 2058 cases. Ann Surg 222: 438-446.

4. Slim H, Tiwari A, Ahmed A, Ritter JC, Zayed H, et al. (2011) Distal versus ultradistal bypass grafts: amputation-free survival and patency rates in patients with critical leg ischaemia. Eur J Vasc Endovasc Surg 42: 83-88.

5. Coppola G, Caro C (2009) Arterial geometry, flow pattern, wall shear and mass transport: potential physiological significance. J R Soc Interface 6: 519-528.

6. Albäck A, Roth WD, Ihlberg L, Biancari F, Lepäntalo M (2000) Preoperative angiographic score and intraoperative flow as predictors of the mid-term patency of infrapopliteal bypass grafts. Eur J Vasc Endovasc Surg 20: 447-453.

7. Methe H, Balcells M, Alegret Mdel C, Santacana M, Molins B, et al. (2007) Vascular bed origin dictates flow pattern regulation of endothelial adhesion molecule expression. Am J Physiol Heart Circ Physiol 292: H2167-2175.

8. Wagner DD, Frenette PS (2008) The vessel wall and its interactions. Blood 111: $5271-5281$

9. Peng X, Haldar S, Deshpande S, Irani K, Kass DA (2003) Wall stiffness suppresses Akt/eNOS and cytoprotection in pulse-perfused endothelium. Hypertension 41: 378-381.

10. Canver CC, Cooler SD, Murray EL, Nichols RD, Heisey DM (1997) Clinica importance of measuring coronary graft flows in the revascularized heart. Ultrasonic or electromagnetic? J Cardiovasc Surg (Torino) 38: 211-215.

11. D'Ancona G, Ricci M, Karamanoukian (2001) Graft patency verification in coronary artery bypass grafting: Principles and clinical application. In: Salerno TA, Ricci M, Karamanoukian HL, D’Ancona G, Bergsland J (eds). Beating Heart Coronary Artery Surgery. Futura Publishing Co. Armonk, NY, USA, pp. 47-56.

12. Ascer E, Veith FJ, Gupta SK, White SA, Bakal CW, et al. (1988) Short vein grafts: a superior option for arterial reconstructions to poor or compromised outflow tracts? J Vasc Surg 7: 370-378.

13. Heise M, Krüger U, Settmacher U, Sklenar S, Neuhaus (1999) A new method of intraoperative hydraulic impedance measurement provides valuable prognostic information about infrainguinal graft patency. J Vasc Surg 30: 301-308.

14. D'Ancona G, Karamanoukian HL, Ricci M, Bergsland J, Salerno TA (2000) Graft patency verification in coronary artery bypass grafting: principles and clinical applications of transit time flow measurement. Angiology 51: 725-731.

15. Johnston KW, Taraschuk I (1976) Validation of the Role of Pulsatility Index in Quantitation of the Severity of Peripheral Arterial Occlusive Disease. Am J Surg 131: 295-297.

16. Gosling RG, Dunbar G, King DH, Newman DL, Side CD, et al. (1971) The quantitative analysis of occlusive peripheral arterial disease by a non-intrusive ultrasonic technique. Angiology 22: 52-55.

17. Morota T, Duhaylongsod FG, Burfeind WR, Huang CT (2002) Intraoperative evaluation of coronary anastomosis by transit-time ultrasonic flow measurement. Ann Thorac Surg 73: 1446-1450.

18. Thul R, Heckenkamp J, Gawenda M, Reichert V, Aleksic M, et al. (2007) The role of intra-operative Prostavasin application during crural bypass surgery. Zentralbl Chir 132: 485-490.

19. Hobbs SD, Pinkney T, Sykes TC, Fox AD, Houghton AD (2009) Patency of infra-inguinal vein grafts--effect of intraoperative Doppler assessment and a graft surveillance program. J Vasc Surg 49: 1452-1458.

20. Rzucidlo EM, Walsh DB, Powell RJ, Zwolak RM, Fillinger MF, et al. (2002) Prediction of early graft failure with intraoperative completion duplex ultrasound scan. J Vasc Surg 36: 975-981. 